\title{
RESECCIÓN TRANSURETRAL DE PRÓSTATA CON BISTURÍ BIPOLAR
}

\author{
R. BARRERO CANDAU*, J.M. SÁNCHEZ ZALABARDO, B. BLASCO BELTRÁN, \\ J. LÁZARO PÉREZ**, J.G. VALDIVIA URÍA
}

Servicio de Urología. **Servicio de Anatomía Patológica. H.C.U. Lozano Blesa. Zaragoza. *Servicio de Urología. H.U. Virgen del Rocío. Sevilla.

Actas Urol Esp. 28 (2): 147-151, 2004

\section{RESUMEN}

RESECCIÓN TRANSURETRAL DE PRÓSTATA CON BISTURÍ BIPOLAR

FUNDAMENTO: Presentar nuestra experiencia inicial en la RTU de próstata con bisturí bipolar axipolar. MATERIAL Y MÉTODO: Los sujetos de estudio han sido 5 pacientes con una edad media de 72 años intervenidos, entre mayo y junio de 2002. Todos presentaban valores elevados en el cuestionario de síntomas (IPSS). El volumen ecográfico medio fue 57,4 g. Se empleó el resector Gyrus ${ }^{\circledR}$ y suero fisiológico para irrigación.

RESULTADOS: La duración media de la intervención fue de 70 minutos. Ningún paciente presentó hiponatremia ni precisó transfusión. En todos los casos se retiró la sonda a las 48 horas, produciéndose una retención aguda de orina (RAO), que precisó sonda dos días más. Todos fueron dados de alta al tercer día, salvo el paciente que presentó el episodio de RAO. Al mes y a 6 meses hay una mejoría mantenida del IPSS.

CONCLUSIONES: La RTU de próstata con bisturí bipolar axipolar puede evitar los efectos secundarios de la reabsorción de glicina y permite abordar próstatas de mayor volumen al disponer de más tiempo para hacerlo. No obstante, hacen falta estudios más amplios para valorar la eficacia de esta nueva tecnología, frente a la RTU de próstata con bisturí monopolar que sigue siendo el patrón oro.

PALABRAS CLAVE: Resección transuretral de próstata. Bipolar. Plasmaquinético.

\section{ABSTRACT}

TRANSURETRAL RESECTION OF PROSTATE WITH BIPOLAR ELECTROBISTURI

FUNDAMENT: To show our initial experience in the TUR of prostate with bipolar axipolar bistoury.

MATERIAL AND METHODS: Five patients with an average age of 72 years old, were operated between may and june 2002. They showed important increased in questionnaire symptoms (IPSS). The average ecografic volume has been $57.4 \mathrm{~g}$. We employed Gyrus ${ }^{\circledR}$ resector and physiological salt solution for continue irrigation.

RESULTS: The average operative time was 70 minutes. None of the patients showed hyponatremia needier blood transfusion. In all the cases the sound was removed 48 hours after operation, one of them have urinary retention (UR) and need sound tow more days. Hospital stay was tree days except the patient how had UR. At 1 and 6 month there is improvement in the IPSS.

CONCLUTIONS: TUR of prostate with bipolar axipolar bistoury can avoid the secondary effects of glicine and allows us to work with prostates of bigger volumes due to we have more time to do it. Nevertheless bigger and better studies are required to value the effectiveness of this new technology opposite the TUR of prostate with monopolar bistoury which keeps being the gold standard.

KEY WORDS: Transuretral resection of prostate. Bipolar. Plasmakinetic. 
$\mathrm{E}$ xiste consenso en que la resección transuretral (RTU) de próstata con bisturí monopolar es el tratamiento estándar de aquellos adenomas de próstata cuyo volumen permita resecarlos en aproximadamente una hora. Sin embargo la RTU de próstata con bisturí monopolar presenta tres inconvenientes claros:

1. El uso de la placa de bisturí (como toma de tierra) para completar el circuito eléctrico hace que la corriente pase a través del paciente produciendo daño del tejido circundante y la posibilidad de producir estimulación nerviosa (Fig. 1).

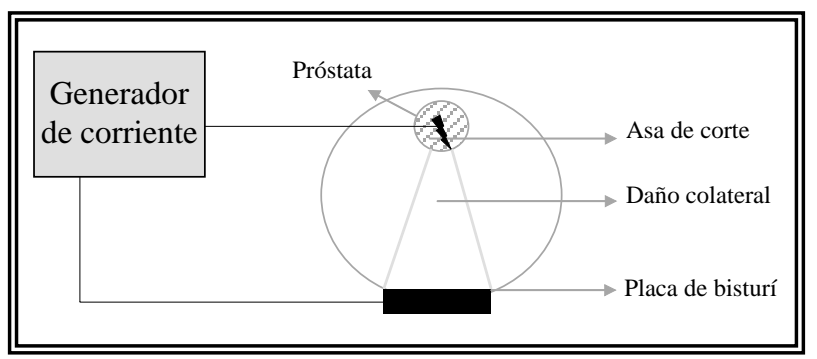

FIGURA 1. Muestra como la utilización del bisturi monopolar clásico además de producir el efecto deseado, crea un paso de corriente a través del paciente produciendo daño del tejido circundante y la posibilidad de desencadenar estimulación nerviosa.

2. El bisturí monopolar no es eficaz para hacer corte y hemostasia al mismo tiempo.

3. La reabsorción de líquidos de lavado no conductores e hiposmolares (como la solución de glicina), puede producir hiponatremia dilucional dando lugar a diversas alteraciones hidroelectrolíticas causantes de alteraciones del ritmo cardíaco, edema cerebral, etc.

A lo largo de la última década han surgido multitud de alternativas mínimamente invasivas encaminadas a la reducción del tejido prostático con menor morbilidad que la RTU. Entre las más relevantes están la criocirugía, la ablación por radiofrecuencia, los ultrasonidos de alta intensidad, la laserterapia intersticial, la termoterapia por microondas y la ablación mediante la inyección de etanol ${ }^{1,2}$. Una de las últimas innovaciones técnicas encaminadas a disminuir la morbilidad de la RTU de próstata es el bisturí bipolar axipolar (sistema plasmakinético). Este trata de evitar los tres problemas principales de la RTU con bisturí monopolar, utilizando la misma técnica quirúrgica ${ }^{3,4}$.
El asa de este tipo de bisturí es similar a las habitualmente utilizadas en la RTU de próstata convencional, de la que se diferencia en que el mismo terminal lleva incluidos el electrodo emisor (asa) y receptor de la corriente, separados ambos por una sección de material de alta resistencia y baja conductancia (Fig. 2). Este bisturí bipolar monoaxial necesita para funcionar que uno de los polos o electrodos esté en contacto con el tejido que se quiera resecar, a diferencia de lo que ocurre con el bisturí bipolar biaxial (ejemplo: Tijera bipolar), que necesita que ambos electrodos estén en contacto con el tejido para su correcto funcionamiento. El bisturí bipolar monoaxial trabaja con suero fisiológico $\mathrm{u}$ otro líquido que haga de conductor entre el electrodo activo y el pasivo. Funciona mediante pulsos de corriente que evitan que se forme un arco eléctrico entre ambos polos mientras que el asa no entre en contacto con el tejido. Una vez que el asa entra en contacto con el tejido la corriente se hace continua circulando desde el electrodo activo (el asa) al electrodo pasivo, a través del medio de menor impedancia o resistencia, es decir, el suero fisiológico. Esta corriente continua hace que el asa alcance una temperatura muy alta, llegando a ponerse incandescente, produciendo la vaporización y necrosis coagulativa del tejido con el que entra en contacto. Gracias a su forma de asa además de vaporizar y producir necrosis coagulativa permite la obtención de fragmentos prostáticos para estudio anatomopatológico ${ }^{3,4}$.

El objetivo de este trabajo es revisar la literatura sobre este tema, para conocer la eficacia de esta innovación técnica, además de presentar nuestros propios puntos de vista tras su utilización.

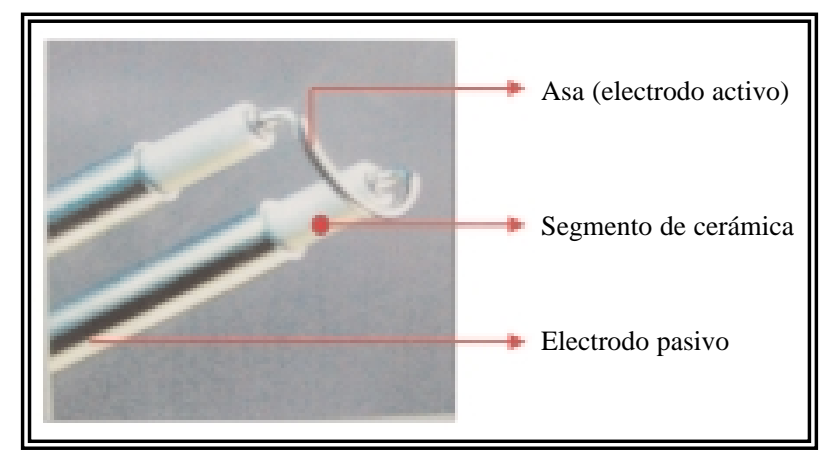

FIGURA 2. Terminal del bisturi bipolar axipolar (sistema plasmaquinético). 


\section{MATERIAL Y MÉTODO}

Los sujetos de estudio han sido 5 pacientes varones con una edad media de 72 años (rango 66-78), intervenidos en el Hospital Clínico Universitario Lozano Blesa de Zaragoza, entre mayo

\section{TABLA I}

CARACTERÍSTICAS DE LOS PACIENTES

\begin{tabular}{||l|c|c|c|c|c||}
\hline Pacientes & $\mathbf{1}$ & $\mathbf{2}$ & $\mathbf{3}$ & $\mathbf{4}$ & $\mathbf{5}$ \\
\hline Edad & 78 & 70 & 69 & 77 & 66 \\
\hline Vol. próstata & 42 & 40 & 45 & 80 & 80 \\
\hline $\begin{array}{l}\text { IPSS } \\
\text { preoperatorio }\end{array}$ & 26 & 23 & 30 & 31 & 32 \\
\hline $\begin{array}{l}\text { Na+ } \\
\text { preoperatorio }\end{array}$ & 144,0 & 144,1 & 139,0 & 141,6 & 139,2 \\
\hline
\end{tabular}
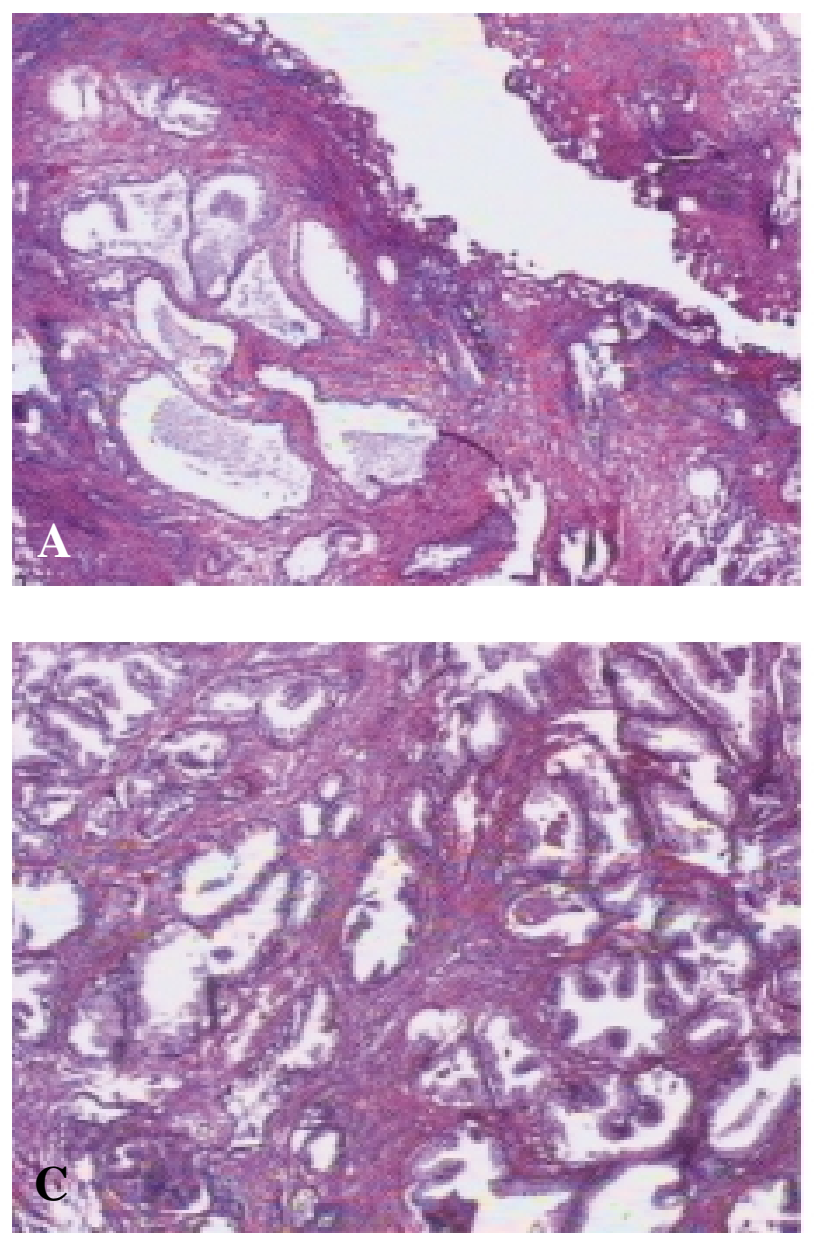

\section{TABLA II}

RESULTADO TRAS LA RTU BIPOLAR

\begin{tabular}{|l|c|c|c|c|c|}
\hline Paciente & $\mathbf{1}$ & $\mathbf{2}$ & $\mathbf{3}$ & $\mathbf{4}$ & $\mathbf{5}$ \\
\hline $\begin{array}{l}\text { Tiempo de } \\
\text { resección }\end{array}$ & 40 & 70 & 65 & 90 & 85 \\
\hline $\begin{array}{l}\text { Na+ } \\
\text { post-operatorio }\end{array}$ & 139,1 & 144,2 & 141,0 & 139,2 & 141,7 \\
\hline $\begin{array}{l}\text { Transfusión } \\
\text { sanguínea }\end{array}$ & No & No & No & No & No \\
\hline $\begin{array}{l}\text { R.A.O. tras } \\
\text { retirar sonda }\end{array}$ & No & No & Sí & No & No \\
\hline \begin{tabular}{l} 
Alta en horas \\
\hline $\begin{array}{l}\text { IPSS a los } 6 \\
\text { meses }\end{array}$
\end{tabular} & 5 & 5 & 8 & 12 & 6 \\
\hline \hline
\end{tabular}
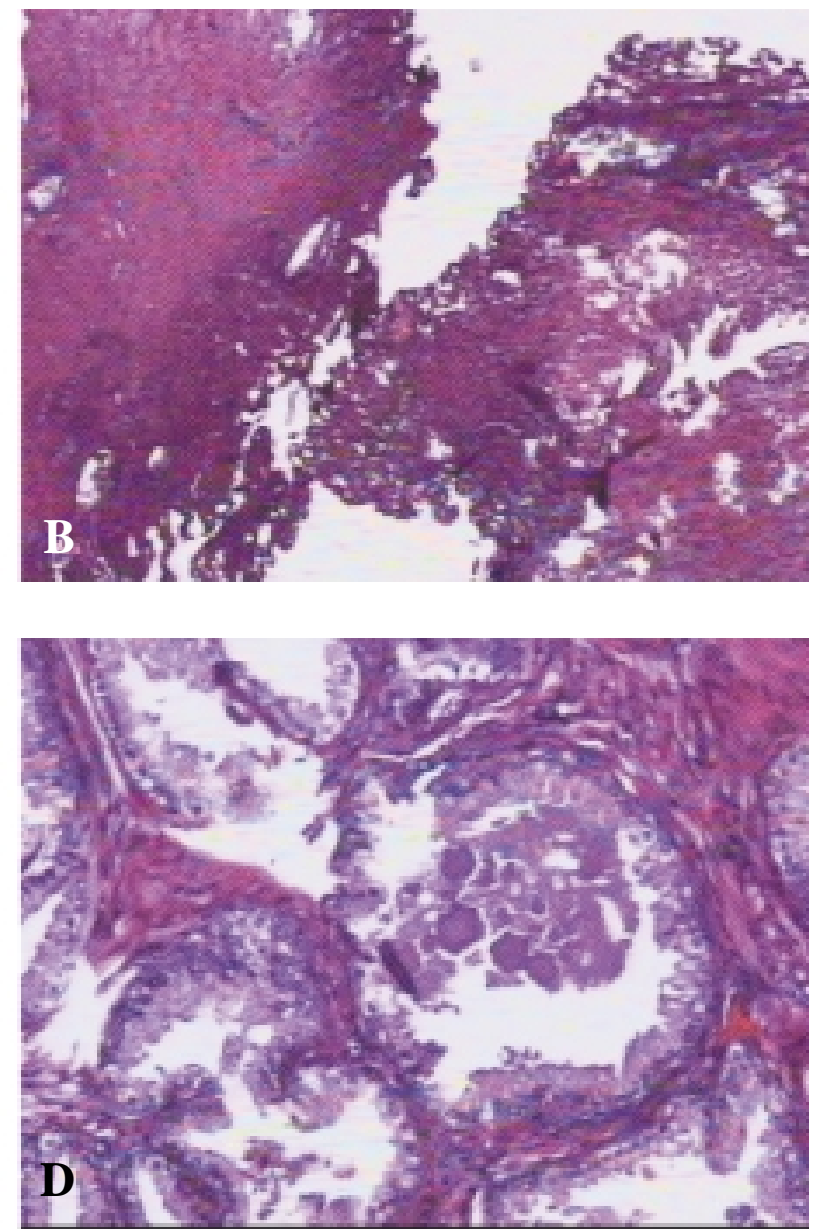

FIGURA 3. En las fotografias A y B se observa la desestructuración de los bordes de los fragmentos resecados, sin embargo (como se puede apreciar en $\mathrm{C}$ y $\mathrm{D}$ ) en el interior de los mismos está conservado el patrón arquitectural glandular, que nos permite su estudio. 
y junio de 2002. Todos ellos presentaban clínica obstructiva del tracto urinario inferior, con puntuaciones altas en los cuestionarios de sintomas (IPSS). Un paciente tenía antecedentes de carcinoma vesical intervenido hace más de 5 años con resección transuretral (RTU) de vejiga, y otro estaba sondado de forma permanente por episodios repetidos de retención aguda de orina. El volumen ecográfico medio, medido en ecografía abdominal, fue de 57,4 g (rango 40-80) (Tabla I).

$\mathrm{El}$ material empleado para las resecciones ha sido el resector axipolar Gyrus ${ }^{\circledR}$ de la casa American Everest, que utiliza un sistema de irrigación succión continua, con un calibre externo $26 \mathrm{Fr}$, óptica de $30^{-}$y un terminal que incluye ambos electrodos, emisor y receptor de la corriente (Fig. 2). Por motivos técnicos, el equipo de esta casa comercial no está compatibilizado aún con ninguna de las casas de endoscopia con las que habitualmente se trabaja y no tiene de momento un sistema para la evacuación de los fragmentos resecados, por lo que al final de la resección utilizamos la camisa $28 \mathrm{Fr}$ del resector de la casa Storz, que acopla con la jeringa de Tomey. El líquido de irrigación utilizado en todos los casos, $\mathrm{y}$ necesario para el correcto funcionamiento de este bisturí, fue suero fisiológico.

Con los pacientes en posición de Lloid Davies y una vez descartada la presencia de neoformaciones vesicales al inicio de la intervención, la sistemática de resección fue la misma en todos los casos, iniciándola con cortes profundos a las 5, las 6 y las 7 horas del huso horario. Inicialmente en las proximidades del cuello vesical y luego avanzando progresivamente hasta verun montanun, completando así la exéresis de la base prostática. Posteriormente se resecaban las partes altas de ambos lóbulos laterales y las masas apicales. Se revisaba hemostasia, la uniformidad de la cápsula y de los contornos del cuello vesical y de la uretra membranosa. Finalmente, se introducía la camisa $28 \mathrm{Fr}$ de la casa Storz y se extraían los fragmentos utilizando una jeringa de Tomey. La cirugía finalizaba dejando sonda foley, acodada, de 3 vías de $22 \mathrm{Fr}$.

\section{RESULTADOS}

La duración media de la intervención fue de 70 minutos (rango 40-90), como se puede obser- var en la Tabla I, a medida que nos hemos familiarizado con el resector hemos abordado próstatas de mayor volumen y utilizado mayor tiempo para hacerlo. El color de la orina al final de la intervención fue claro en todos los casos y como se puede observar en la Tabla II, ningún paciente precisó transfusión. No se produjeron hiponatremias y en todos los casos la sonda se retiró a las 48 horas de la intervención, produciéndose en el sujeto 3, un episodio de retención aguda de orina (RAO), que precisó sonda durante dos días más, tras los cuales se retiró y orinó espontáneamente. Todos fueron dados de alta al tercer día, salvo el paciente que presentó el episodio de retención que fue dado de alta dos días más tarde. En las revisiones post-cirugía, al mes y a los 6 meses, hay una mejoría clínica mantenida al comparar los IPSS pre y post-intervención (Tablas I y II).

\section{DISCUSIÓN}

Hay dos series prospectivas, sin controles ni aleatorización, pero con resultados concordantes entre ellas ${ }^{5,6}$, que muestran una disminución en la puntuación de los cuestionarios de sintomas y mejoría de la flujometría tras este tipo de resección. En estos artículos comentan los autores la correcta hemostasia, sin que fuera necesaria transfusión en ningún caso, y la ausencia de alteraciones hidroelectrolíticas. Según lo publicado tras el análisis de estas series, esta nueva tecnología tiene una serie de ventajas como son: utilizar suero fisiológico en vez de glicina, que permite alargar el tiempo de resección y abordar próstatas de mayor tamaño, sin que exista riesgo de producir alteraciones hidroelectrolíticas ni de sangrado importante. También se sugiere que producen menor daño perilesional, al no circular la corriente a través del enfermo. En estos trabajos se ha conseguido una disminución de la estancia hospitalaria, incluso en uno de ellos la resección se realizó en un programa de cirugía mayor ambulatoria, siendo dados de alta en el día, el $85 \%$ de los pacientes, que regresaban a las 48 horas para la retirada de sonda ${ }^{5}$.

En nuestra corta experiencia con este electrobisturí, no comercializado aún, hemos podido comprobar la buena hemostasia del mismo, alargando el tiempo de resección sin que se produjeran alteraciones clínicas ni analíticas. Nuestra 
estancia hospitalaria es menor en los enfermos en los que hemos utilizado este sistema. Pero, sin embargo, hemos podido observar la dificultad que presenta para cortar en superficies sobre las que ya se ha cortado, probablemente debido a la desecación del tejido que se produce al cortar vaporizando, como hace este bisturí.

Sin embargo esta innovación técnica, al igual que el resto de alternativas mínimamente invasivas, tiene el inconveniente de que la experiencia hasta el momento es corta y no existen estudios que lo comparen con la RTU monopolar. Aún no hay evidencias que demuestren estas ventajas, aunque sí buenos resultados preliminares, concordantes entre las dos series publicadas, y comprobados en nuestros propios pacientes.

Otro de los inconvenientes que pensamos podía plantear este bisturí era que al cortar por vaporización del tejido, podría dar fragmentos no útiles para su estudio anatomopatológico. Tras el estudio microscópico de los fragmentos prostáticos resecados (Fig. 3), hemos podido observar la conservación de la arquitectura glandular prostática, lo que permite un estudio histológico fiable.

\section{CONCLUSIONES}

La RTU de próstata con bisturí bipolar puede evitar los efectos secundarios de la reabsorción de glicina, ya que utiliza suero fisiológico, que no produce alteraciones clínicas ni hidroelectrolíticas. Esto permite abordar próstatas de mayor volumen al disponer de más tiempo para hacerlo, pues el límite de tiempo impuesto por la posibilidad de reabsorción de glicina desaparece. No obstante, hacen falta estudios prospectivos, controlados, aleatorizados y con un seguimiento amplio para valorar la eficacia de esta nueva tecnología, frente a la RTU de próstata con bisturí monopolar que sigue siendo el patrón oro.

\section{REFERENCIAS}

1. JEPSEN JV, BRUSKEWITH RC.: Recent developments in the surgical management of benign prostatic hyperplasia. Urology 1998; 51: 23-31.

2. NAKADE SY, JOHNSON DB.: Ablación tisular invasiva con agujas y no invasiva. AUA update serie 2001; 3: 75-82.

3. http: //www.gyrusmedical.com

4. http: //www.jnjgateway.com/home.jhtml

5. EATON AC, FRANCIS RN.: The provision of transurethral prostatectomy on a day-case basis using bipolar plasma kinetic technology. B J U Int 2002; 89: 534-537.

6. BOTTO H, LEBRET T, BARRE P, ORSONI JL, HERVE JM, LUGAGNE PM.: Electro vaporization of the prostate with the gyrus device. J Endourol 2001; 15: $313-316$

Dr. R. Barrero Candau

C/ Castillo Alcalá de Guadaira, 17

Portal C-2, $1^{\circ}$ A

41013 Sevilla

(Trabajo recibido el 7 febrero de 2003) 